\title{
ANDES

www.scielo.cl

\section{Adrenoleucodistrofia ligada a X: Un caso de presentación aguda cerebral infantil}

\author{
X-linked adrenoleukodystrophy: A case of acute childhood cerebral presentation
}

\author{
Sebastián Posada Bustos ${ }^{\mathrm{a}}$, Marco Luciano Charry Lopez ${ }^{\mathrm{b}}$, Eugenia Espinosa García ${ }^{\mathrm{c}}$
}

aResidente Neurología Pediátrica, Universidad Militar Nueva Granada. Hospital Militar Central. Bogotá, Colombia

bServicio de Neurorradiología, Hospital Militar Central. Bogotá, Colombia

‘Servicio de Neurología Pediátrica, Universidad Militar Nueva Granada. Hospital Militar Central. Bogotá, Colombia

Recibido: 23 de septiembre de 2020; Aceptado: 7 de enero de 2021

¿Qué se sabe del tema que trata este estudio?

La adrenoleucodistrofia ligada al X (ADLX) es un trastorno peroxisomal que produce acumulación de ácidos grasos de cadena muy larga en sistema nervioso central que conlleva a desmielinización cerebral progresiva que conduce a una paraplejia espástica, insuficiencia suprarrenal y deterioro cognitivo.
¿Qué aporta este estudio a lo ya conocido?

Paciente con una ADLX de presentación cerebral rápidamente progresiva, asociado a insuficiencia suprarrenal, con desmielinización progresiva por resonancia de cerebro en tres meses, con una mutación de novo en la familia, no candidato a trasplante de medula ósea.

\section{Resumen}

La adrenoleucodistrofia ligada al cromosoma X (ADLX) es una enfermedad peroxisomal, debida a la mutación en el gen ABCD1 que conllevan acumulo de ácidos grasos de cadena muy larga en los tejidos. Objetivo: Describir el caso de un paciente con ADLX de presentación cerebral infantil aguda, revisar su proceso diagnóstico y posibilidades terapéuticas. Caso Clínico: Escolar masculino de 7 años, con cuadro clínico de seis meses de evolución de disminución en la agudeza visual, dificultades escolares por inatención, alteración en lectoescritura y aislamiento social. Al examen físico, disminución bilateral de agudeza visual de rápida progresión, hipoprosexia, lesiones hiperpigmentadas en las manos, e inestabilidad para la marcha. Estudio inicial de resonancia magnética cerebral (RMC) mostró alteración de la señal de sustancia blanca parieto-occipital bilateral, índice de compromiso neurorradiologico de Loes de 12 puntos. Se documentó insuficiencia adrenal, cumpliendo criterios clínicos de ADLX. Se realizó estudio de ácidos grasos de cadena muy larga, encontrándose elevados, confirmándose el diagnóstico. Tres meses más tarde evoluciona a pérdida de la visión e incapacidad para la marcha, se realizó RMC con un índice de Loes de 15 puntos debido a compromiso extenso estructural de sistema nervioso central (SNC), deterioro rápidamente progresivo, por lo que no se consideró candidato para trasplante de medula ósea. Conclusión: El presente caso de ADLX es una forma de presentación cerebral infantil aguda, de rápida progresión. La evaluación clínica y clasificación de hallazgos de compromiso radiológico por índice de Loes, deben guiar la elección para decidir el manejo.
Palabras clave:

Adrenoleucodistrofia; Desmielinización; Insuficiencia Adrenal; Ácidos Grasos 


\begin{abstract}
$\mathrm{X}$-linked adrenoleukodystrophy (X-ALD) is a peroxisomal disease due to a mutation in the ABCD1 gene that leads to the accumulation of very-long-chain fatty acids in tissues. Objective: To describe one patient with severe childhood cerebral X-ALD and to analyze his diagnostic process and therapeutic possibilities. Clinical Case: 7 -year-old male child, with a six-month history of decreased visual acuity, learning difficulties due to lack of attention, reading and writing impairment, and social isolation. On physical examination, he presented bilateral decrease in visual acuity, hypoprosexia, hyperpigmented lesions on the hands, and gait abnormality. Brain MRI showed bilateral white matter signal alteration in parieto-occipital regions, with 12 points on the Loes' scale. He also presented adrenal insufficiency, meeting clinical criteria for X-ALD. Very-long-chain fatty acid was elevated, confirming the diagnosis. Three months later, the patient progressed to vision loss and inability to walk. MRI was repeated showing 15 points in the Loes' scale due to extensive structural involvement of the central nervous system, with rapidly progressive deterioration. Therefore, he was not considered a candidate for bone marrow transplantation. Conclusion: This case of X-ALD was of severe childhood cerebral presentation, with rapid progression. The clinical evaluation and classification of radiological findings according to the Loes' scale should guide the choice of management.
\end{abstract}

Keywords:

X-linked

Adrenoleukodystrophy;

Demyelination;

Adrenal Insufficiency;

Fatty Acids

\section{Introducción}

La adrenoleucodistrofia ligada a X (ADLX), es una enfermedad neurodegenrativa peroxisomal causada por la mutación del gen $\mathrm{ABCD} 1$, que se configura como la leucodistrofia más común en el mundo, con alta variabilidad de expresión clínica, incluso en una misma familia ${ }^{1}$. El espectro clínico puede variar desde una forma cerebral progresiva, que lleva a discapacidad severa en la primera década de vida, pasando por una adrenoleucomielopatía de inicio en la adultez, o una presentación de enfermedad de Addison ${ }^{2}$. El objetivo de esta comunicación es presentar un caso de ADLX de presentación cerebral infantil, que tuvo una rápida progresión de los síntomas.

\section{Caso Clínico}

Escolar masculino de 7 años, producto de primer embarazo controlado, hijo de padres no consanguíneos, previamente sano, con neurodesarrollo acorde para su edad y rendimiento escolar adecuado hasta su primer año de escuela. Debutó con cuadro de progresión rápida, que inició con alteración de agudeza de visual asociado a exotropía izquierda. Dos meses después presentó dificultades escolares dadas por inatención, dificultades en seguimiento de instrucciones, con pérdida de algunos logros aprendidos en lectura y cálculo básico. A los 4 meses presentó dificultades de audición, con necesidad de repetición de las instrucciones para realización de las tareas, labilidad emocional, aislamiento social y pobre interés en las relaciones sociales que notan los profesores. A los 6 meses de evolución se evidenció caídas frecuentes, y necesidad de marcha con apoyo en lugares que no conoce, por aumento de compromiso visual, lo que lo llevó a desescolarización.

$\mathrm{Al}$ examen neurológico, alerta, con dificultad en seguimiento de instrucciones, hipoprosexia, con dificultades en calculo y lectura, así como en abstracción y analogías para su edad, con una agudeza visual bilateral de 20/150, Rinne positivo, sin alteración de otros pares craneales, fuerza y sensibilidad sin alteraciones, normorreflexico, con marcha con aumento del polígono de sustentación, sin ataxia. Ante este cuadro de regresión del desarrollo aguda se consideraron diagnósticos diferenciales (tabla 1). Como hallazgo llamativo se observó hiperpigmentación en los nudillos de las manos y encías, lo que hizo sospechar ADLX.

Se realizó estudio con Resonancia Magnética de cerebro con espectroscopia que evidenció en secuencia T2 hiperintensidad de la sustancia blanca de predominio profundo parietoccipital bilateral y temporal posterior con compromiso del esplenio del cuerpo calloso y tractos corticoespinales sin restricción de la difusión y curvas de espectroscopia con elevación de colina, $\mathrm{n}$ acetil-aspartato y pico de lactato lo que hacía sospechar como primera posibilidad adrenoleucodistrofia ligada a X (figuras 1 y 2 ).

Se realizaron exámenes de laboratorio que descartaron alteración electrolítica. Presentó niveles ACTH cinco veces por sobre los valores normales, que indicaban insuficiencia adrenal, por el momento asintomática. Se inició suplencia con Hidrocortisona. Se solicitó perfil de ácidos grasos de cadena muy larga, encontrándose elevación del ácido tetracosanoico (C24) y hexacosanoico (C26), con relaciones C24/C22 y C26/ C22 elevadas, alteración en la función discriminativa para hombres y mujeres con la enfermedad peroxisomal, de acuerdo con estudios previos ${ }^{3}$, lo que fue com- 
Tabla 1. Posibles diagnósticos diferenciales a considerar en casos de regresión del desarrollo en escolares

Adrenoleucodistrofia ligada a X
Deficit pantotenato kinasa
Encefalopatías mitocondriales
Enfermedad de Huntington
Enfermedad de Krabbe
Enfermedad de Wilson
Esclerosis múltiple
Gangliosidosis tipo II
Leucodistrofia metacromática
Lipofuscinosis ceroide neuronal - juvenil
Panencefalitis esclerosante subaguda
Sialidosis forma juvenil
Tumor cerebral

patible con adrenoleucodistrofia ligada a X. Se realizó estudio genético con secuenciación completa de gen ABCD1, identificándose que es portador hemicigoto de la variante patogénica c.900+1G > A, que confirmó diagnóstico a nivel molecular. Su cuadro clínico correspondió a una presentación rápidamente progresiva de la infancia.

Con la confirmación del diagnóstico, se realizó evaluación de las imágenes de resonancia magnética, para cuantificar el grado de compromiso del sistema nerviosos central, de acuerdo a la escala de Loes ${ }^{4}$, concluyendo puntaje 12 puntos.

El paciente evolucionó tres meses después con pérdida de la visión e incapacidad en la marcha indepependiente. Se repitió Resonancia Magnética de cerebro, que mostraba aumento de compromiso neurorradiologico con un índice de Loes de 15 puntos y tractografia que muestra marcada alteración de la anisotropia los tractos de la sustancia blanca en regiones posteriores (figura 3).

Teniendo en cuenta que la única terapia que ha mostrado beneficio en retraso de la enfermedad es el trasplante de medula ósea, en pacientes con deterioro neurológico leve o nulo, con escala de Loes menor a 9
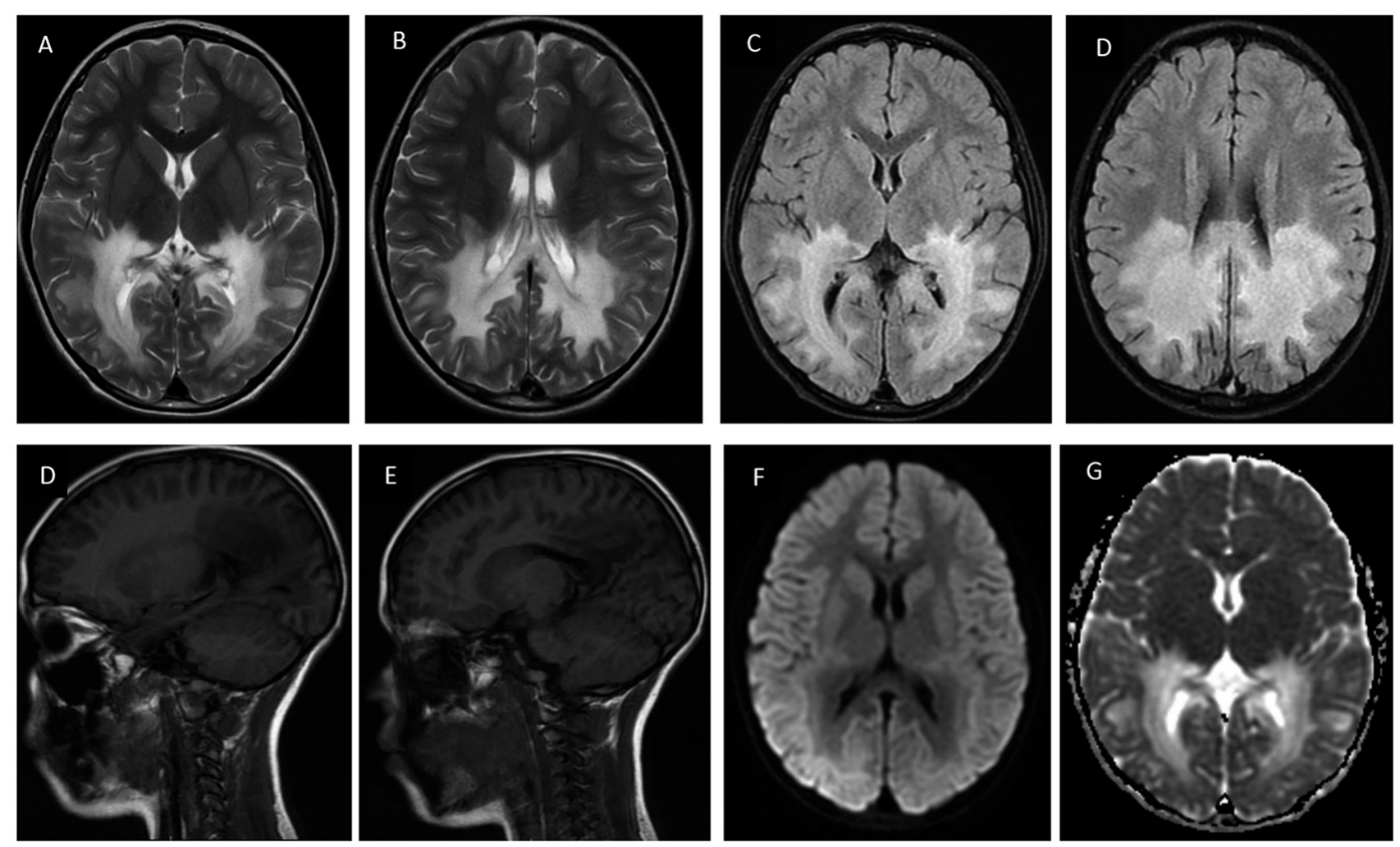

Figura 1. Resonancia Magnética cerebral. A y B: secuencia T2, C y D: secuencia T2 FLAIR. D y E: secuencia T1. Se observa hiperintensidades en secuencias T2 e hipointensidades en secuencia T1 en sustancia blanca parieto-occipital bilateral y temporal posterior con compromiso del esplenio del cuerpo calloso. F: Difusión y G: ADC. Se observa restricción de la Difusión y aumento de la intensidad en ADC en sustancia blanca parietooccipital bilateral. 
puntos, y sin déficit cognitivo, se concluyó que el paciente no es candidato a la realización de trasplante de medula ósea, ya que sus beneficios son menores a sus posibles complicaciones y se inició el manejo de apoyo, asesoría genética y seguimiento interdisciplinario.

El paciente evolucionó al año con deterioro neurológico, espasticidad generalizada, trastorno de la deglución severo y estado vegetativo aparente.

\section{Discusión}

La ADLX es el trastorno peroxisomal más común, presente en todas las regiones del mundo. Es una enfermedad rara en la población pediátrica, con una incidencia de una incidencia estimada de 1 en 17000 a 35000 recién nacidos ${ }^{5,6}$.

Se caracteriza por alteración de la beta-oxidación peroxisomal de ácidos grasos de cadena muy larga (VLCFA; $\geq$ C22) acumulándose en todos los tejidos, incluyendo la sustancia blanca del cerebro, médula espinal y corteza suprarrenal. Está causada por mutaciones en el gen $\mathrm{ABCD} 1$ localizado en el cromosoma $\mathrm{X}^{7}$. En el caso del paciente se encontró dicha elevación del ácido tetracosanoico (C24) y hexacosanoico (C26), con relaciones C24/C22 y C26/C22 elevadas.

En la actualidad aún no están dilucidadas las bases moleculares para la heterogeneidad clínica de la $\mathrm{ADLX}^{8}$. No existe una correlación genotipo-fenotipo general. Todos los pacientes tienen mutación en el gen que codifica para el transportador de casete de unión a ATP peroxisomal (ABCD1), que produce la proteína ALDP (proteína de la adrenoleucodistrofia), cuya función es transportar ácidos grasos de cadena muy larga activados añadiéndoles coenzima A del citosol hacia el peroxisoma para su degradación? .

Esta enfermedad tiene una forma de herencia ligada a X, con cerca del $4 \%$ de mutaciones son de novo. Se han descrito más de 800 tipos de mutaciones, que se encuentran consignadas en la base de datos disponible en línea (www.x-ald.nl) ${ }^{6,10}$. En el caso del paciente presentado no existe historia familiar de la enfermedad. Fue confirmado como portador hemicigoto de la variante patogénica c. $900+1 \mathrm{G}>\mathrm{A}$, que se asocia niveles reducidos de la proteína ALDP, mecanismo causal de la enfermedad.

Como se mencionado, la presentación clínica de la ADLX es variable. El caso presentado corresponde a la presentación cerebral, con un fenotipo rápidamente progresivo y devastador ${ }^{5}$. En la infancia la clínica se caracteriza por un inicio insidioso, con déficit en las habilidades cognitivas, que involucran las funciones visoespaciales, visomotoras, atención y razonamiento, así como pueden presentase con un comportamiento hiperactivo o retraído, ejemplificado en el caso ${ }^{11}$. La
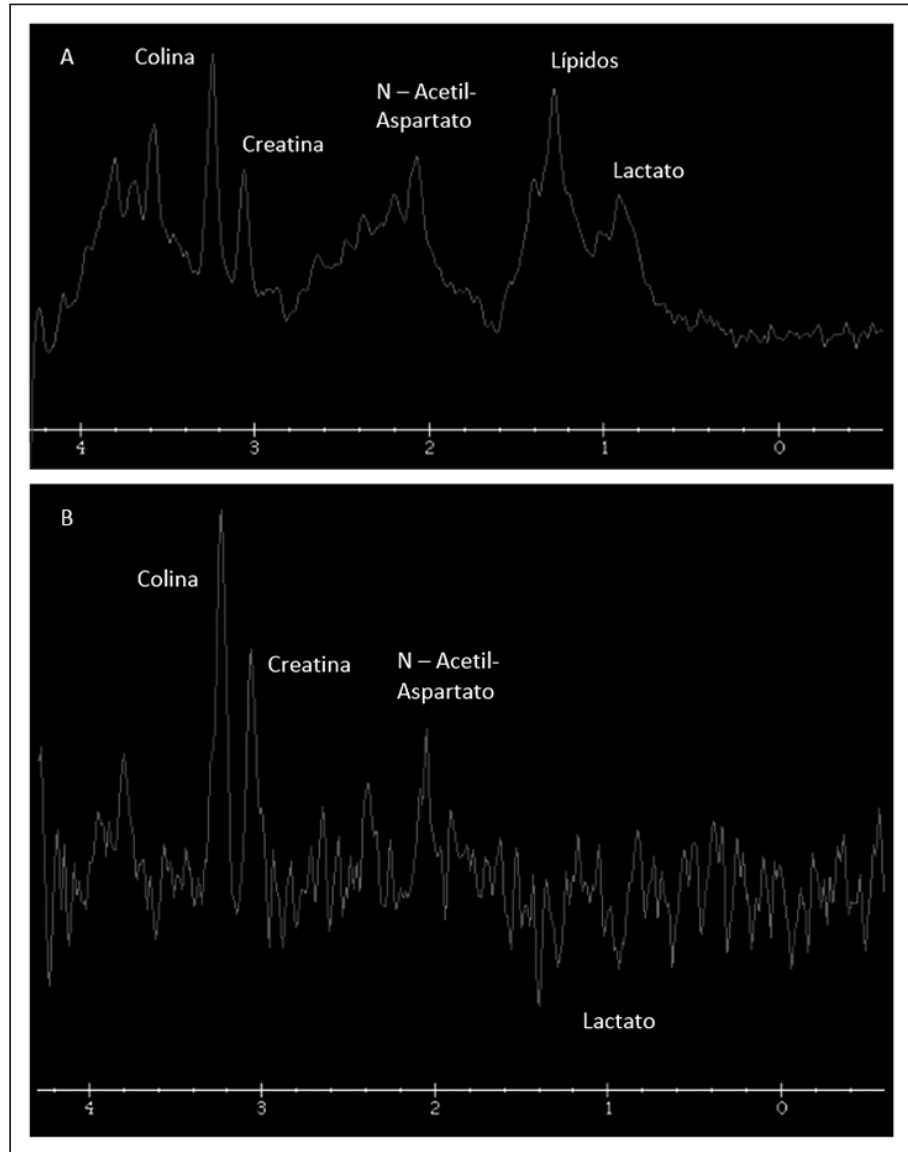

Figura 2. Espectroscopia por resonancia magnética cerebral univoxel A: Tiempo de eco corto: Se observa leve aumento de Colina y N-AcetilAspartato, con elevación de Lactato. B: Tiempo de eco medio: Se observa inversión del peak de Lactato.

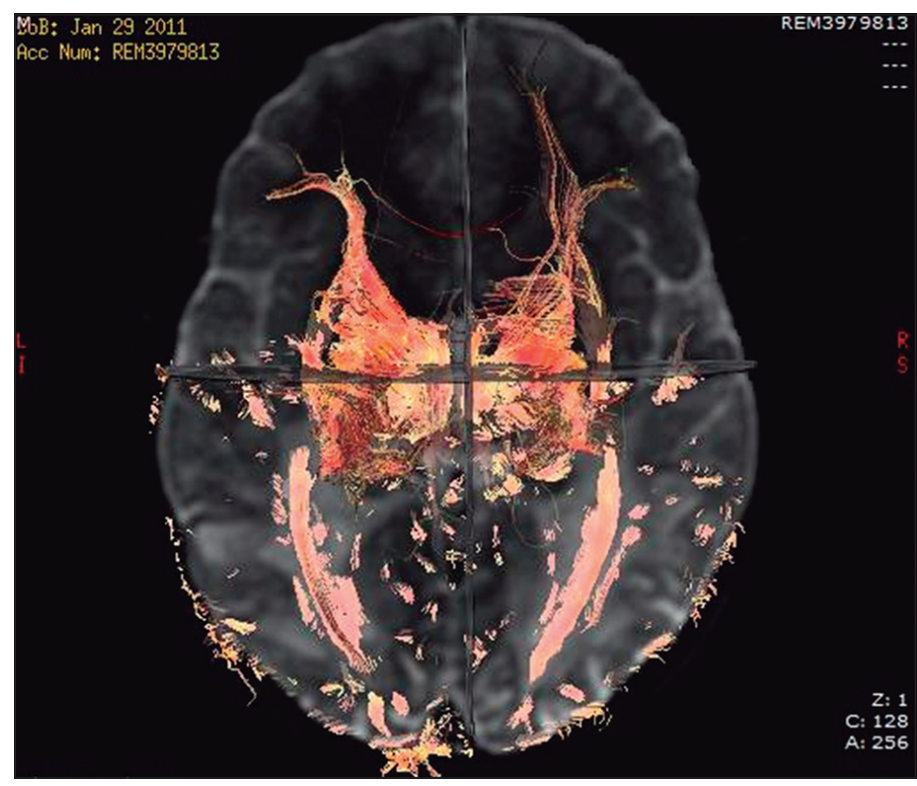

Figura 3. Reconstrucción de la vía óptica por tractografia por resonancia magnética. Confirma marcada alteración de sustancia blanca posterior con alteración de fascículos que conforman radiaciones ópticas a ese nivel. 
manifestación inicial puede ser una disminución en el rendimiento académico, y se puede catalogar como trastorno del aprendizaje o déficit de atención e hiperactividad lo que podría retrasar el diagnóstico, como sucede en el caso presentado. En todo paciente pediátrico con deterioro del rendimiento escolar y/o pérdida de habilidades adquiridas se debe sospechar una enfermedad neurodegenerativa, sobretodo ante la ausencia de historia de trastorno del neurodesarrollo previo (tabla 1).

En la medida en la que la enfermedad progresa aparecen nuevos déficits neurológicos, como apraxia, astereognosia, alteración auditiva, disminución de la agudeza visual, hemiparesia, ataxia o crisis epilépticas $^{11}$. En el caso presentado, al momento de la consulta ya presentaba alteración de la agudeza visual y alteración auditiva, lo que muestra el rápido avance clínico. El compromiso avanza hasta la incapacidad de entender el lenguaje, ceguera cortical, imposibilidad para la deambulación, incapacidad para la comunicación, necesidad de alimentación por sonda orogástrica $o$ gastrostomía, evolución hacia estado vegetativo, y se describe que la muerte ocurre en estos pacientes dos a cuatro años luego del inicio de los síntomas ${ }^{5,6}$. En el caso del paciente en tres meses el niño tuvo importante deterioro visual y pérdida de la marcha, y al año de inicio de la enfermedad se encuentra en estado vegetativo.

El compromiso cerebral se inicia en el 80\% de los pacientes en el esplenio del cuerpo calloso y progresa envolviendo la sustancia blanca adyacente parietooccipital $^{12}$. Las lesiones inicialmente afectan los tractos piramidales en el puente o la capsula interna y se extienden a la sustancia blanca del centro semioval ${ }^{12}$. La Resonancia Magnética de cerebro muestra intensidades anormales (aumentadas en T2 y FLAIR y disminuidas en T1), en el cuerpo calloso, sustancia blanca parieto-occipital o frontal, tracto piramidal dentro del tallo, puente y capsula interna ${ }^{5}$. La Resonancia siempre es anormal en el compromiso cerebral, y estos hallazgos mencionados junto a la clínica configuran una alta sospecha de la enfermedad, como el caso presentado, que tenía compromiso bilateral simétrico de la sustancia blanca parieto-occipital, con compromiso del esplenio del cuerpo calloso y tractos corticoespinales.

En 1994 Loes propuso una escala de puntaje de acuerdo al compromiso cerebral por Resonancia Magnética que permite medir el grado de afectación cerebral y que esta aceptada para medir el progreso de la enfermedad, así como criterio de evaluación para definir los mejores candidatos para acceder a trasplante de medula ósea ${ }^{4}$. Estudios previos muestran correlación entre el compromiso estructural por Resonancia Magnética y pronóstico, lo que hace de esta imagen imprescindible para el diagnóstico y seguimiento de estos pacientes ${ }^{13}$.
La insuficiencia adrenal primaria es una característica clínica muy importante en los pacientes con ADLX. Se caracteriza por niveles elevados de ACTH y bajos niveles de cortisol, los pacientes pueden presentar hiperpigmentación y tiene una presentación variable en los pacientes entre el 50 y el $100 \%{ }^{6}$. En un estudio prospectivo de una cohorte de niños neurológicamente pre-sintomáticos, se encontró que el $80 \%$ tenían evidencia bioquímica de insuficiencia adrenal silente $^{14}$, lo que ejemplifica la importancia de buscar y tratar en conjunto con Endocrinología los pacientes con insuficiencia adrenal, teniendo en cuenta de que puede ser el primer hallazgo de la enfermedad. Inclusive existe un fenotipo de esta enfermedad que sólo se manifiesta con insuficiencia adrenal primaria ${ }^{15,16}$. En el caso descrito, el paciente presentaba hallazgos bioquímicos de insuficiencia adrenal, y solo presentaba discreta hiperpigmentación en las manos y episodios de alteración del estado de conciencia autolimitados y vómito, por lo que se inició tratamiento con corticoides de inmediato.

El diagnóstico de ADLX se confirma por exámenes bioquímicos o examen genético, como se presenta en el caso. Si un hombre cursa con una clínica clara e insuficiencia adrenal, el hallazgo de ácidos grasos de cadena muy larga elevados en plasma confirma el diagnóstico ${ }^{6}$. En mujeres con adrenoleucodistrofia, entre el 10 y el 15\% tienen niveles de VLCFA normales, por lo que se recomienda realizar estudio genético de la mutación $\mathrm{ABCD} 1^{18}$.

Por ahora en nuestro medio el único tratamiento que ha mostrado retrasar su progresión es el trasplante de medula ósea alogénico, y se sabe que los pacientes que se benefician de esta terapia son aquellos con síntomas leves o asintomáticos neurológicos, con una escala de Loes de compromiso por Resonancia Magnética de cerebro menor a 10 puntos y sin alteración cognitiva ${ }^{18}$. En una serie de pacientes chinos se encontró que la realización del trasplante en paciente con enfermedad cerebral avanzada podía acelerar más su progresión ${ }^{19}$. En nuestro paciente la familia solicitaba tratamiento curativo, lo que representa un reto para el grupo clínico en esta enfermedad neurodegenerativa. Se evaluó la posibilidad de realizar el trasplante. Sin embargo, dado que presentó un fenotipo rápidamente progresivo, con alto compromiso neurológico, un puntaje de Loes mayor a 10 y disfunción cognitiva, y teniendo en cuenta además que la mortalidad derivada del trasplante y sus complicaciones es alta ${ }^{18}$, se descartó esta posibilidad.

El manejo con trasplante de médula ósea permanece en discusión. En los pacientes con criterios clínicos adecuados para el trasplante, en un seguimiento a largo plazo de 62 pacientes, $67 \%$ presentaron déficit neurocognitivo severo, mostrando incluso que el mayor beneficio se encontraba en pacientes con punta- 
je de Loes entre 0.5 y 4, que corresponde a pacientes cuyo diagnóstico se hace por tamizaje prenatal o por historia familiar ${ }^{20}$. Actualmente surgen interrogantes, acerca de que si la única posibilidad de instaurar un tratamiento efectivo con resultados realmente buenos, sea realizando tamizaje neonatal, lo que daría una luz de tratamiento a pacientes como el presentado ${ }^{21}$.

Se han estudiado otras opciones terapéuticas. El aceite de Lorenzo, basado en la administración de ácidos grasos C18 y C22, no alteran la concentración de VLCFA en sistema nervioso central y no mejoran la función endocrinológica o neurológica en pacientes con adrenoleucodistrofia ${ }^{22}$. La lovastatina, tampoco mostro reducción en los niveles de VLCFA, así como tampoco impactó en las variables clínicas ${ }^{23}$. Por último, se ha avanzado en el desarrollo de terapia génica con lentivirus, que ha mostrado ser segura, pero tiene pendiente los estudios de seguimiento para evaluar su resultado clínico ${ }^{24}$.

\section{Conclusión}

La ADLX es la enfermedad peroxisomal más frecuente, de presentación clínica variable. Presentamos un paciente con una forma cerebral rápidamente progresiva, sin historia familiar, que inicio con alteraciones de la agudeza visual y dificultades de aprendizaje, con imágenes típicas de esta enfermedad, a nivel bioquímico con insuficiencia adrenal, y con estudio confirmatorio con ácidos grasos de cadena muy larga elevados y estudio genético compatible, en que el trasnplante de médula ósea se descartó como opción de manejo dado el compromiso neurológico.

\section{Responsabilidades Éticas}

Protección de personas y animales: Los autores declaran que los procedimientos seguidos se conformaron a las normas éticas del comité de experimentación humana responsable y de acuerdo con la Asociación Médica Mundial y la Declaración de Helsinki.

Confidencialidad de los datos: Los autores declaran que han seguido los protocolos de su centro de trabajo sobre la publicación de datos de pacientes.

\section{Derecho a la privacidad y consentimiento informa-} do: Los autores han obtenido el consentimiento informado de los pacientes y/o sujetos referidos en el artículo. Este documento obra en poder del autor de correspondencia.

\section{Conflicto de intereses}

Los autores declaran no tener conflicto de intereses.

\section{Referencias}

1. Cappa M, Bizzarri C, Vollono C, et al. Adrenoleukodystrophy. Endocr Dev. 2011;20:149-60.

2. Pereira F, Matte U, Habekost C, et al. Mutations, Clinical Findings and Survival Estimates in South American Patients with X-Linked Adrenoleukodystrophy. PLoS ONE. 2012;7(3):e34195.

3. Moser A, Kreiter N, Bezman L, et al. Plasma very long chain fatty acids in 3,000 peroxisome disease patients and 29,000 controls. Ann Neurol. 1999;45(1):100-10.

4. Loes DJ, Hite S, Moser H, et al. Adrenoleukodystrophy: a scoring method for brain MR observations. AJNR Am J Neuroradiol. 1994;15(9):1761-6.

5. Kemp S, Huffnagel I, Linthorst G, et al. Adrenoleukodystrophy - neuroendocrine pathogenesis and redefinition of natural history. Nat Rev Endocrinol. 2016;12(10):606-15.

6. Jardim LB, da Silva AC, Blank D, et al. X linked adrenoleukodystrophy: clinical course and minimal incidence in South Brazil. Brain Dev. 2010;32(3):180-90.

7. Engelen M, Kemp S, de Visser M, et al.
X-linked adrenoleukodystrophy (X-ALD): clinical presentation and guidelines for diagnosis, follow-up and management. Orphanet J Rare Dis. 2012;7(1):51.

8. Berger J, Forss-Petter S, Eichler FS. Pathophysiology of X-linked adrenoleukodystrophy. Biochimie. 2014; 98:135-42.

9. Engelen M, Kemp S, Poll-The B. X-Linked Adrenoleukodystrophy: Pathogenesis and Treatment. Curr Neurol Neurosci Rep. 2014;14(10):486.

10. Wang Y, Busin R, Reeves C, et al. X-linked adrenoleukodystrophy: ABCD1 de novo mutations and mosaicism. Mol Genet Metab. 2011;104(1-2):160-6.

11. Moser HW, Smith KD, Watkins PA, et al. In The Metabolic and Molecular Bases of Inherited Disease. 8th edition. Edited by Scriver CR, Beaudet AL, Sly WS, Valle D. New York: McGraw Hill; 2001:3257301.

12. Van der Knaap MS, Valk J: X-linked adrenoleukodystrophy. In Magnetic Resonance of Myelination and Myelin Disorders. 3rd edition. Edited by Heilmann U. Berlin-Heidelberg-New York: Springer; 2005:176-90.
13. Moser HW, Loes DJ, Melhem ER, et al. X-Linked adrenoleukodystrophy: overview and prognosis as a function of age and brain magnetic resonance imaging abnormality. A study involving 372 patients. Neuropediatrics. 2000;31(5):227-39.

14. Dubey P, Raymond GV, Moser AB, et al. Adrenal insufficiency in asymptomatic adrenoleukodystrophy patients identified by very long-chain fatty acid screening. J Pediatr. 2005;146(4):528-32.

15. Hsieh S, White PC: Presentation of primary adrenal insufficiency in childhood. J Clin Endocrinol Metab 2011;96:E925-E928.

16. Nascimento M, Rodrigues N, Espada F, et al. Adrenoleukodystrophy: a forgotten diagnosis in children with primary Addison's disease. BMJ Case Rep. 2012;15 .

17. Valianpour F, Selhorst JJ, van Lint LE, et al. Analysis of very long-chain fatty acids using electrospray ionization mass spectrometry. Mol Genet Metab. 2003;79(3):189-96.

18. Miller WP, Rothman SM, Nascene D, et al. Outcomes after 
allogeneic hematopoietic cell transplantation for childhood cerebral adrenoleukodystrophy: the largest single-institution cohort report. Blood. 2011;118(7):1971-8.

19. Jiang MY, Cai YN, Liang CL, et al. Clinical, biochemical, neuroimaging and molecular findings of $\mathrm{X}$-linked Adrenoleukodystrophy patients in South China. Metab Brain Dis. 2015;30(6):143944.

20. Pierpont EI, Eisengart JB, Shanley R, et al. Neurocognitive Trajectory of Boys Who Received a Hematopoietic Stem Cell Transplant at an Early Stage of Childhood Cerebral Adrenoleukodystrophy. JAMA Neurol. 2017;74(6):710-7.

21. Van Haren K, Engelen M. Decision Making in Adrenoleukodystrophy. JAMA Neurol. 2017;74(6):641.

22. van Geel BM, Assies J, Haverkort EB, et al. Progression of abnormalities in adrenomyeloneuropathy and neurologically asymptomatic X-linked adrenoleukodystrophy despite treatment with “Lorenzo's oil”. J Neurol Neurosurg Psychiatry. 1999;67(3):290-9.

23. Engelen M, Ofman R, Dijkgraaf $\mathrm{MG}$, et al. Lovastatin in X-linked adrenoleukodystrophy. N Engl J Med. 2010;362(3):276-7.

24. Eichler F, Duncan C, Musolino PL, y col. Terapia génica de células madre hematopoyéticas para la adrenoleucodistrofia cerebral. N Engl J Med. 2017;377(17):1630-38. 